Ein Tag Notfalldienst ...

Nach dem Aufruf durch Prof. Hans Stalder, Genf, im Namen der Kommission «Recherche et réalisation en médecine appliquée» (RRMA) der SAMW, die jeden Text mit Fr. 500.- vergütet, erschienen in der Schweizerischen Ärztezeitung Nr. 10 vom 6. März 2002, publizieren wir hier weitere Texte. Informationen erhalten Sie unter folgender E-Mail-Adresse: hans.stalder@hcuge.ch.

Un jour de garde...

A la suite de l'invitation par le Professeur Hans Stalder, Genève, au nom de la Commission Recherche et réalisation en médecine appliquée RRMA de l'ASSM, qui rétribue les textes par Fr. 500.-, parue dans le Bulletin des médecins suisses no 10 du 6 mars 2002 , nous publions d'autres textes. Des informations peuvent être obtenues en écrivant à l'adresse e-mail suivante: hans.stalder@hcuge.ch.

\title{
Sechs iatrogene Notfälle
}

\author{
B. Horn
}

Die folgenden sechs iatrogenen Notfälle haben sich glücklicherweise nicht an einem einzigen Tag ereignet. Immerhin könnte die eine oder andere Erkenntnis geeignet sein, da und dort Konsequenzen zu ziehen oder doch mindestens über die menschliche Unzulänglichkeit zu lächeln und sich immer wieder bewusst zu sein, dass es nebst solidem Wissen, Können und sozialen Kompetenzen auch einwenig Glück braucht. Da es grundsätzlich darum geht, aus Fehlern zu lernen, sollte die koordinierte Einführung eines medizinischen Critical-Incident-Reporting-Systems (CIRS medical) dringend unterstützt werden.

\section{Schock nach Schlangenbiss}

Praktikant in einem kleinen Krankenhaus im Berner Oberland. Ein 14jähriger Bub mit einem Schlangenbiss (Kreuzotter) ist angemeldet. Der Junge ist ansprechbar, blass, mit kaltem Schweiss auf der Stirne, Puls 120 pro Minute, BD 70/40 $\mathrm{mm} \mathrm{Hg}$. Ich versuche eine «Leitung» zu legen, Venenverweilkanülen gab es nicht, die dicke, bereits x-mal aufsterilisierte Kanüle findet in der Ellenbeuge eine Vene, und ich lasse $\mathrm{NaCl}$ im Strahl einfliessen. Zwei Sekunden später sehe ich eben den Luft-Flüssigkeit-Spiegel in der Vene verschwinden. Ein Schlauch voll Luft im Kreislauf! Ein Engramm aus der Lenggenhager-Vorlesung blitzt auf, nun kommt Betrieb in den kleinen Notfallraum, sogar die Oberin legt ihren Apfel beiseite. Der Bub wird in maximale Kopftief-Beine-hoch-Lage verbracht. Sehr bange Minuten vergehen, der Patient beginnt zu reklamieren. Nach 10 Minuten allmähliche Normallagerung, es passiert nichts. Nach einer Stunde geht des dem Buben gut, und die Klasse kann die Schulreise mit zwei Stunden Verspätung fortsetzen. Kurzbericht an den Hausarzt.

\section{Fazit}

- Luft im Kreislauf ist gefährlich.

- Handgriffe (Skills, Fertigkeiten) müssen drillmässig geübt sein.

- Ungeübte Ärzte sind gefährlicher als Giftschlangen.

\section{Atemstillstand nach Procain-Penicillin- Injektion}

Kurz vor dem Staatsexamen, erster Tag meiner ersten Praxisvertretung. Am Nachmittag hat mich der Praxisinhaber exakt in mein Pflichtenheft eingeführt, wir haben alle Problempatienten besprochen und zehn Hausbesuche gemacht. Stabübergabe um 18 Uhr, um 19 Uhr beginnt die Abendsprechstunde - allein. Es sitzen zwölf Patienten im Wartezimmer. Der erste Patient ein Koloss aus der Schwerindustrie - kann kaum sprechen («hot potatoe voice»), er hat eine hochfebrile eitrige Angina.

Ich lege den Mann hin, ziehe eine Ampulle Procain-Penicillin auf und injiziere es - nach gründlichem Aspirieren - ventroglutäal. Nach einer Minute lasse ich den Patienten aufstehen und sich anziehen, spüle die (Glas-Metall-)Spritze am Lavabo, dann knallt es. Der Koloss liegt bewusstlos am Boden, schnappt zwei Male nach Luft und wird blau. Ich renne ins Wartezimmer und rufe drei starke Männer um Hilfe, spritze eine halbe Ampulle Adrenalin in die Kopfhaut («The Ship captains medical book»), beginne sofort mit 
Beatmen (Puls hatte der Mann immer), der Garagist des Städtchens macht den Wagen bereit, zwei Handwerker tragen, ich beatme und ab geht es in routiniert-rasender Fahrt in Richtung Kantonsspital. Kurz nach der Einlieferung beginnt der nun intubierte Patient zu atmen, am nächsten Tag wird er das Spital verlassen können. Wir fahren zurück in die Praxis, und ich lade den Garagisten zu einem Bier in der «Sonne» ein. Daraus wird nichts: Im Wartezimmer sitzen immer noch die zehn zurückgelassenen Patienten und harren auf den Doktor, der da kommen soll. Das fast grenzenlose Vertrauen in einen jungen Nochnicht-ganz-Arzt wirft mich fast um. Als die Sprechstunde fertig ist, ist die «Sonne» geschlossen ... Nach einer Woche kommt der Koloss zur Kontrolle und dankt für die gute Behandlung ...

\section{Fazit}

- Reanimation muss drillmässig geübt werden.

- Was den Patienten letztendlich gerettet hat (Adrenalin? Beatmung? beides?), wissen wir nicht.

- Das Vertrauen der Bevölkerung in die Ärzte ist immer noch gross, daran gerüttelt wird in erster Linie von den Medien.

\section{Asystolie nach Verapamil intravenös}

Eine ältere Frau ruft mich wegen einer Attacke von Herzklopfen. Ich fahre hin, die Frau liegt in der finsteren Stube im Bett, Puls regelmässig, 160 pro Minute, BD 130/80 mm Hg, die Patientin hat Angst und Harndrang. Das EKG bestätigt den Verdacht auf eine paroxysmale supraventrikuläre Tachykardie. Mehrmaliges ValsalvaManöver (Eiswasser, tief einatmen, pressen) und die Carotis-Sinus-Massage sind erfolglos. Die Patientin will nicht ins Spital, der Leidensdruck ist hoch, das Herz nicht mehr jung, ich entschliesse mich zur damals üblichen Verabreichung von Verapamil i.v. durch eine Venenverweilkanüle. Wenige Sekunden nach der (langsamen!) Injektion verdreht die Frau die Augen, sie wird blassgrau, ist komatös, weder Puls noch BD sind messbar. Das EKG (bei noch liegenden Elektroden) zeigt eine Asystolie. Einige kräftige Schläge aufs Präkordium bleiben ohne Erfolg. Telefon ans Spital «sofort Ambulanz mit Rea-Equipe an B-Gasse 32». Ich zerre die Frau auf den Fussboden, verabreiche 1 Ampulle Atropin i.v. und beginne mit Beatmung und Herzmassage «EinhelferMethode». Von weitem höre ich das rasch lauter werdende Martinshorn. Eine gut eingespielte Rettungswagenequipe ist für den Hausarzt (und Patienten ...) Gold wert.
Unterdessen hat sich eine «weak action» eingestellt, dann ein Kammerflimmern, das defibrilliert wird, die Frau überlebt, ohne Hirnschaden, und lebt noch mehrere Jahre in guter Lebensqualität.

\section{Fazit}

- Kenntnis und Anwendung wichtiger Nebenwirkungen/Interaktionen.

- Der Hausarzt ist oft grässlich allein.

- Selten lässt sich ein Kreislaufstillstand sogar zu Hause beheben.

\section{Waschpulveringestion}

Der zweijährige Sohn ist am Sonntagmorgen auffallend ruhig. Ich finde ihn, in der Waschküche am Boden sitzend mehr oder weniger lustvoll Waschpulver «Floris» und «Maga» essend. Floris enthält Borsäure, also sofort ins nahe Spital. Der Internist ist auf der IPS beschäftigt, der Chirurg (wie immer) im Ops. Also nichts wie los, selbst ist der Mann, problemloses Einführen der Magensonde, Kontrolle der Lage und ganz vorsichtiges Einlaufenlassen von wenig kaltem Wasser. Trotz aller Vorsicht entwickelt sich sofort ein gewaltiger Schaum (was immerhin die Waschpulveringestion beweist ...), und nach 5 Minuten ist der Sohn blaugrau. Die Schwester holt im Ops die Anästhesistin, ich sinke auf einen Stuhl und sehe bereits die BLICK-Schlagzeile vor mir: «Jungarzt tötet Sohn bei Magenspülung». Nach einer Viertelstunde ist der Junior wieder rosig, und der Vater erholt sich.

\section{Fazit}

- Lebhafte Kinder, die ruhig sind, müssen hellhörig machen.

- Kinder stecken alles in den Mund, auch wenn es noch so scheusslich schmeckt.

- Aktionsradius des Kindes = doppelte Armlänge.

- Vorsicht bei Behandlung von Angehörigen, Objektivität schwierig.

- Magenspülung ohne Intubation kann tödlich enden.

\section{Hypotonie und Oligurie nach ACE-Hemmer-Testdosis}

Der 80jährige Landwirt leidet an einem gemischten Aortenvitium. Bereits vor Jahren hat er eine invasive Abklärung und Therapie verweigert, und er lässt sich trotz zunehmender Dyspnoe nicht umstimmen. Der Kardiologe 
empfiehlt einen ACE-Hemmer, und der Patient erhält die Hälfte der niedrigsten Dosierung in der Praxis ( $1 / 2$ Tabl. Lopirin 12,5 mg). Nach einer Stunde beträgt der BD noch 90/60 mm Hg, dann ist während mehrerer Stunden kein BD messbar, der Patient verweigert aber eine Spitaleinweisung strikte. An den folgenden Tagen kommt es zu einer Oligurie und zu einem massiven Anstieg des Kreatinins. ACE-Hemmer müssen wir vergessen. Ein älterer Kollege empfiehlt mir einen Versuch mit Morphintropfen. Mit diesen lebt der Patient noch mehrere Jahre (!!) bei guter Lebensqualität. Er stirbt nach akuter Verschlechterung unter dem Bild einer kardialen Kachexie (Endocarditis marantica?).

\section{Fazit}

- Der Wille des Patienten ist oberstes Gesetz.

- Schwer Herzkranke vertragen ACE-Hemmer oft schlecht.

- Sogenannte «uralte» Pharmaka können einem Patienten oft helfen.

\section{Digitalisintoxikation}

Die 85jährige Engländerin verbringt seit Jahren ihre Ferien in der Jungfrauregion. Die nette Dame mit spitzenbesetzten weissen Handschuhen sitzt unangemeldet im Wartezimmer wegen Herzklopfen und Atemnot. Check-up in England vor zwei Wochen OK. Der Puls beträgt 140/Minute mit absoluter Arrhythmie, Pulsdefizit 60/Minute! Deutliche Lungenstauung. Die dezidierte Dame lehnt eine Spitaleinweisung ab. Ich gebe ihr in der Praxis 3 Tabl. Digoxin 0,25 und notiere auf der Schachtel «every morning 1». Nach zwei Tagen ruft mich der Hotelier, der Dame gehe es schlecht, sie erbreche und könne kaum aufstehen. Ich fahre sofort hin. Puls 36/Minute, arrhythmisch, BD 90/70 mm Hg, das EKG zeigt einen totalen AV-Block und polytope ventrikuläre Ersatzsystolen. Ich frage, ob sie die Tabletten exakt genommen habe. «Sure, every morning seven». Tempestas! Die Frau hat meine 1 als 7 gelesen und innert 48 Stunden 17 Tabletten Digoxin 0,25 geschluckt, eine schwere Digitalisvergiftung. Die Frau verbringt drei Tage auf der Intensivstation und kommt sich anschliessend für die Behandlung bedanken ...

\section{Fazit}

- Einheitliche 1 und 7 in Europa wären eine vornehme EU-Aufgabe.

- Hat der Patient/die Patientin mich sicher richtig verstanden?

\section{Aus Fehlern lernen oder: «aus Schaden wird man klug»}

Der letzte tödliche Schlangenbiss in der Schweiz hat sich vor über 30 Jahren ereignet. Wie viele Menschen in dieser Zeit medizinischen Aktivitäten zum Opfer gefallen sind, wissen wir nicht, sicher sind es nicht jährlich dreitausend, wie eine aus den USA hochgerechnete (zweifelhafte) Studie, die durch das Bundesamt für Sozialversicherung (BSV) verbreitet wurde, wahrhaben wollte. Immerhin spielen sich trotz guter Aus-, Weiter- und Fortbildung, guter Organisation und optimaler Medikamentensicherheit immer wieder schwerwiegende medizinische Pannen ab, die zu folgenschweren Schäden oder gar Todesfällen führen können. Dramatische Notfälle in der Praxis sind relativ selten, iatrogene (zum Glück) noch seltener, aber nicht ausgeschlossen. Die Bestrebungen vorab der Anästhesisten, auch in der Schweiz ein Meldesystem für iatrogene «Pannen» in der Medizin einzurichten, müssen mit allen Mitteln unterstützt werden. CIRS (Critical Incident Reporting System) nennt sich das Projekt, meiner Ansicht nach eine gute Sache. Das Know-how konnte von den Fluggesellschaften bezogen werden, die «BeinaheKatastrophen» seit Jahren mit Akribie sammeln, auswerten und daraus Konsequenzen ziehen. Die ambulante Medizin, speziell auch die Grundversorger, sollte sich an solchen Studien zur Verbesserung der Versorgungsqualität unbedingt mitbeteiligen, da sonst eines Tages «Guidelines» ohne Beizug von Praktikern publiziert werden könnten, die nur bedingt oder überhaupt nicht praxistauglich sind. Scheuen wir uns deshalb nicht vor begangenen Fehlern und unterstützen wir geeignete Datenbanken. Nur wer nichts tut, macht auch keine Fehler, es geht nicht um Bestrafung, sondern es geht darum, aus medizinischen Pannen Konsequenzen zu ziehen. 